\title{
Finite groups with some nonnormal subgroups of non-prime-power order
}

\author{
Jiangtao ShI, Cui Zhang and Dengfeng LiAng
}

(Received May 16, 2013; Revised October 6, 2013)

\begin{abstract}
We prove that a group $G$ with exactly three classes of nonnormal proper subgroups of the same non-prime-power order is nonsolvable if and only if $G \cong A_{5}$, and a group $G$ with exactly four classes of nonnormal proper subgroups of the same nonprime-power order is nonsolvable if and only if $G \cong P S L(2,7)$ or $P S L(2,8)$. Moreover, we prove that any group $G$ with at most nine classes of nonnormal nontrivial subgroups of the same order is always solvable except for $G \cong A_{5}, P S L_{2}(7)$ or $S L_{2}(5)$.

Key words: nonsolvable group, class of the same order, nonnormal, non-prime-power order.
\end{abstract}

\section{Introduction}

In this paper all groups are considered to be finite. In [1], Gallian and Moulton gave a complete classification of minimal non-prime-power order groups. As a direct corollary of [2, Theorems 4.2 and 4.4], any group $G$ with at most three conjugacy classes of proper subgroups of non-prime-power order is always solvable except for $G \cong A_{5}$. Note that any two conjugate subgroups must have the same order but any two subgroups of the same order might not be conjugate. As a direct corollary of [3, Theorems 3.1 and 3.2], the following result holds:

Theorem 1.1 Any group with at most two classes of proper subgroups of the same non-prime-power order is always solvable, and a group $G$ with exactly three classes of proper subgroups of the same non-prime-power order is nonsolvable if and only if $G \cong A_{5}$.

In [4], we extended the above results:

2010 Mathematics Subject Classification : 20D05, 20 D10.

The first author was partially supported by NSFC (Grant 11201401, 11361075 and 11561021).

The second author was partially supported by NSFC (Grant 11201403 and 11561021) and research program P1-0285 from the Slovenian Research Agency.

The third author was supported by Research Foundation for Youth Scholars of Beijing Technology and Busines University (QNJJ2011-50). 
Theorem 1.2 ([4]) A group $G$ with exactly four conjugacy classes of proper subgroups of non-prime-power order is nonsolvable if and only if $G \cong P S L_{2}(8)$, and a group $G$ with exactly four classes of proper subgroups of the same non-prime-power order is nonsolvable if and only if $G \cong P S L_{2}(7)$ or $P S L_{2}(8)$.

In order to further extend the above results, we only consider the nonnormal proper subgroups of non-prime-power order. [5, Lemma 3.1] shows that any group with at most two classes of nonnormal nonnilpotent proper subgroups of the same order is always solvable. As a direct corollary of [5, Lemma 3.1], the following result holds:

Theorem 1.3 Any group with at most two classes of nonnormal proper subgroups of the same non-prime-power order is always solvable.

In this paper, our main results are as follows, the proofs of which are given in Section 2 and Section 3 respectively.

Theorem 1.4 Suppose that $G$ is a nonsolvable group with exactly three classes of nonnormal proper subgroups of the same non-prime-power order, then $G \cong A_{5}$.

Theorem 1.5 Suppose that $G$ is a nonsolvable group with exactly four classes of nonnormal proper subgroups of the same non-prime-power order, then $G \cong P S L_{2}(7)$ or $P S L_{2}(8)$.

By considering the number of classes of nonnilpotent proper subgroups of the same order and the number of classes of nontrivial nilpotent subgroups of the same order in [3], and by considering the number of classes of proper subgroups of the same non-prime-power order and the number of classes of nontrivial subgroups of the same prime-power order in [4], we all obtained the following result.

Theorem 1.6 ([3], [4]) Let $G$ be a group.

(1) Suppose that $G$ has at most six classes of nontrivial subgroups of the same order, then $G$ is solvable.

(2) Suppose that $G$ is a nonsolvable group with exactly seven classes of nontrivial subgroups of the same order, then $G \cong A_{5}$.

(3) Suppose that $G$ has exactly eight classes of nontrivial subgroups of the same order, then $G$ is solvable. 
(4) Suppose that $G$ is a nonsolvable group with exactly nine classes of nontrivial subgroups of the same order, then $G \cong P S L_{2}(7)$.

As an extension of Theorem 1.6, only consider the number of classes of nonnormal nontrivial subgroups of the same order, we have the following result, the proof of which is given in Section 4 .

Theorem 1.7 Let $G$ be a group.

(1) Suppose that $G$ has at most six classes of nonnormal nontrivial subgroups of the same order, then $G$ is solvable.

(2) Suppose that $G$ is a nonsolvable group with exactly seven classes of nonnormal nontrivial subgroups of the same order, then $G \cong A_{5}$.

(3) Suppose that $G$ has exactly eight classes of nonnormal nontrivial subgroups of the same order, then $G$ is solvable.

(4) Suppose that $G$ is a nonsolvable group with exactly nine classes of nonnormal nontrivial subgroups of the same order, then $G \cong P S L_{2}(7)$ or $S L_{2}(5)$.

\section{Proof of Theorem $\mathbf{1 . 4}$}

Lemma 2.1 Suppose that $G$ is a nonsolvable group with exactly three classes of nonnormal nonnilpotent proper subgroups of the same order, then $G \cong A_{5}$ or $S L_{2}(5)$.

Proof. Let $H$ be a minimal nonsolvable subgroup of $G$. Then $H / \Phi(H)$ is a minimal nonabelian simple group. It is easy to see that $H$ has no normal nonnilpotent proper subgroups. By the hypothesis and [3, Theorem 3.1], $H$ has exactly three classes of nonnilpotent proper subgroups of the same order. Therefore, by [3, Theorem 3.2], one has $H \cong A_{5}$ or $S L_{2}(5)$.

We claim that $G=H$.

Otherwise, assume that $H<G$. By the hypothesis, one must have $H \unlhd G$. It follows that $\Phi(H) \unlhd G$ since $\Phi(H)$ char $H$. Consider the quotient group $G / \Phi(H)$. Let $N / \Phi(H)$ be a subgroup of $G / \Phi(H)$ such that $H / \Phi(H)$ is a maximal subgroup of $N / \Phi(H)$. Let $H / \Phi(H)=\bar{H}$ and $N / \Phi(H)=\bar{N}$. Obviously, $\bar{N} / \bar{H}$ is a cyclic group of prime order. Assume that $\bar{N} / \bar{H} \cong \mathbb{Z}_{p}$ for some prime $p$. Obviously, $C_{\bar{N}}(\bar{H}) \cap \bar{H} \unlhd \bar{H}$. Since $\bar{H} \cong A_{5}$ is a nonabelian simple group, we have $C_{\bar{N}}(\bar{H}) \cap \bar{H}=1$. By N/C-theorem, $\bar{N} / C_{\bar{N}}(\bar{H})=$ $N_{\bar{N}}(\bar{H}) / C_{\bar{N}}(\bar{H}) \lesssim \operatorname{Aut}(\bar{H})=\operatorname{Aut}\left(A_{5}\right)=S_{5}$. 
(a) Assume that $C_{\bar{N}}(\bar{H})=1$. Since $\bar{N}>\bar{H} \cong A_{5}$, it follows that $\bar{N} \cong S_{5}$.

(b) Assume that $C_{\bar{N}}(\bar{H}) \neq 1$. It follows that $\bar{N}=C_{\bar{N}}(\bar{H}) \times \bar{H}$. Therefore, $C_{\bar{N}}(\bar{H}) \cong C_{\bar{N}}(\bar{H}) \bar{H} / \bar{H}=\bar{N} / \bar{H} \cong \mathbb{Z}_{p}$. One has $\bar{N}=C_{\bar{N}}(\bar{H}) \times \bar{H} \cong$ $\mathbb{Z}_{p} \times A_{5}$.

If $\bar{N} \cong S_{5}$ or $\mathbb{Z}_{p} \times A_{5}$. Then $\bar{N}$ has more than three classes of nonnormal nonnilpotent proper subgroups of the same order. It follows that $N$ has more than three classes of nonnormal nonnilpotent proper subgroups of the same order, a contradiction.

Thus we have $G=H$. It follows that $G \cong A_{5}$ or $S L_{2}(5)$.

Proof of Theorem 1.4. As a direct corollary of Lemma 2.1, we have $G \cong$ $A_{5}$.

\section{Proof of Theorem $\mathbf{1 . 5}$}

Proof. Let $K$ be a minimal nonsolvable subgroup of $G$. One has that $K / \Phi(K)$ is a minimal nonabelian simple group.

Assume that $\Phi(K)$ has non-prime-power order. Then for every proper subgroup $H$ of $K$ such that $H>\Phi(K), H$ is a nonnormal proper subgroup of $K$ of non-prime-power order. Since any nonsolvable group has at least seven classes of nontrivial subgroups of the same order by Theorem 1.6, it follows that $K$ has at least seven classes of nonnormal proper subgroups of the same non-prime-power order, a contradiction.

Thus $\Phi(K)$ has prime-power order. It is easy to see that $K$ has no normal proper subgroups of non-prime-power order. By the hypothesis and Theorem 1.1, we have that $K$ has exactly three or four classes of proper subgroups of the same non-prime-power order.

Assume that $K$ has exactly three classes of proper subgroups of the same non-prime-power order. By Theorem 1.1, $K \cong A_{5}$. Since $G$ has exactly four classes of nonnormal proper subgroups of the same non-prime-power order and $A_{5}$ has exactly three classes of nonnormal proper subgroups of the same non-prime-power order, one has $K<G$. Let $M$ be a subgroup of $G$ such that $K$ is a maximal subgroup of $M$. If $K \unlhd M$, one has $M \cong S_{5}$ or $A_{5} \times \mathbb{Z}_{p}$ for some prime $p$. It is easy to see that both $S_{5}$ and $A_{5} \times \mathbb{Z}_{p}$ have more than four classes of nonnormal proper subgroups of the same non-prime-power order, it follows that $M$ has more than four classes of nonnormal proper subgroups of the same non-prime-power order, a contradiction. Next assume that $K$ is 
not normal in $M$. If $M$ has no normal proper subgroups of non-prime-power order, then $M$ has exactly four classes of proper subgroups of the same nonprime-power order. By Theorem 1.2, one has $M \cong P S L(2,7)$ or $P S L(2,8)$. Obviously, $A_{5}$ cannot be a maximal subgroup of $P S L(2,7)$ or $P S L(2,8)$, a contradiction. If $M$ has a normal proper subgroup of non-prime-power order, say $T$. Obviously, $T \cap K=1$. It follows that $M=T \rtimes K \cong T \rtimes A_{5}$. For every nontrivial subgroup $A$ of $K, T \rtimes A$ is a nonnormal proper subgroup of $M$ of non-prime-power order. It follows that $M$ has more than four classes of nonnormal proper subgroups of the same non-prime-power order, also a contradiction.

Thus $K$ has exactly four classes of proper subgroups of the same nonprime-power order. By Theorem 1.2, one has $K \cong P S L_{2}(7)$ or $P S L_{2}(8)$.

We claim that $G=K$.

Otherwise, assume that $K<G$. Let $N$ be a subgroup of $G$ such that $K$ is a maximal subgroup of $N$. By the hypothesis, one must have $K \unlhd N$. Obviously, $C_{N}(K) \cap K \unlhd K$. Since $K \cong P S L_{2}(7)$ or $P S L_{2}(8)$ is a nonabelian simple group, one has $C_{N}(K) \cap K=1$. By N/C-theorem, $N / C_{N}(K)=$ $N_{N}(K) / C_{N}(K) \lesssim \operatorname{Aut}(K)=P G L(2,7)$ or $P \Gamma L(2,8)$.

(i) Assume that $C_{N}(K)=1$. Since $K$ is a maximal subgroup of $N$, it follows that $N \cong P G L(2,7)$ or $P \Gamma L(2,8)$.

(ii) Assume that $C_{N}(K) \neq 1$. It follows that $N=C_{N}(K) \times K$. Therefore, $C_{N}(K) \cong C_{N}(K) K / K=N / K \cong \mathbb{Z}_{p}$. One has $N=C_{N}(K) \times K \cong$ $\mathbb{Z}_{p} \times P S L_{2}(7)$ or $\mathbb{Z}_{p} \times P S L_{2}(8)$.

Since $P G L(2,7), P \Gamma L(2,8), \mathbb{Z}_{p} \times P S L_{2}(7)$ and $\mathbb{Z}_{p} \times P S L_{2}(8)$ have more than four classes of nonnormal proper subgroups of the same non-primepower order, it follows that $N$ has more than four classes of nonnormal proper subgroups of the same non-prime-power order, a contradiction.

Thus we have $G=K \cong P S L_{2}(7)$ or $P S L_{2}(8)$.

\section{Proof of Theorem 1.7}

Lemma 4.1 Any group $G$ with at most three classes of nonnormal nontrivial subgroups of the same prime-power order is solvable.

Proof. Assume that $G$ is nonsolvable. Let $G$ be a counterexample of minimal order. It follows that $G$ is a minimal nonsolvable group. Then $G / \Phi(G)$ is a minimal nonabelian simple group. Obviously, $G / \Phi(G)$ has at least 
four classes of nontrivial subgroups of the same prime-power order. Since $G / \Phi(G)$ is a nonabelian simple group, it follows that $G$ has at least four classes of nonnormal nontrivial subgroups of the same prime-power order, a contradiction. Therefore, the counterexample does not exist and so $G$ is solvable.

The proof of Theorem 1.7 follows from the following two lemmas.

Lemma 4.2 Let $G$ be a group.

(1) Suppose that $G$ has at most six classes of nonnormal nontrivial subgroups of the same order, then $G$ is solvable.

(2) Suppose that $G$ is a nonsolvable group with exactly seven classes of nonnormal nontrivial subgroups of the same order, then $G \cong A_{5}$.

(3) Suppose that $G$ has exactly eight classes of nonnormal nontrivial subgroups of the same order, then $G$ is solvable.

Proof. By $\tau_{1}(G)$ we denote the number of classes of nonnormal nontrivial subgroups of $G$ of the same prime-power order and by $\tau_{2}(G)$ we denote the number of classes of nonnormal proper subgroups of $G$ of the same nonprime-power order. Then $G$ has exactly $\tau_{1}(G)+\tau_{2}(G)$ classes of nonnormal nontrivial subgroups of the same order. If $G$ is nonsolvable, by Lemma 4.1 and Theorem 1.3, one has $\tau_{1}(G) \geq 4$ and $\tau_{2}(G) \geq 3$.

(1) Suppose that $\tau_{1}(G)+\tau_{2}(G) \leq 6$. One must have either $\tau_{1}(G) \leq 3$ or $\tau_{2}(G) \leq 2$. Then $G$ is solvable.

(2) Suppose that $G$ is a nonsolvable group with $\tau_{1}(G)+\tau_{2}(G)=7$. One must have $\left(\tau_{1}(G), \tau_{2}(G)\right)=(4,3)$. By Theorem $1.4, G \cong A_{5}$.

(3) Suppose that $\tau_{1}(G)+\tau_{2}(G)=8$. Assume that $G$ is nonsolvable. One must have $\left(\tau_{1}(G), \tau_{2}(G)\right)=(5,3)$ or $(4,4)$. If $\left(\tau_{1}(G), \tau_{2}(G)\right)=(5,3)$. By Theorem 1.4, $G \cong A_{5}$. However, $\tau_{1}\left(A_{5}\right)=4 \neq 5$, a contradiction. If

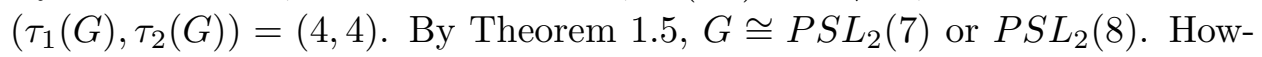
ever, $\tau_{1}\left(P S L_{2}(7)\right)=5 \neq 4$ and $\tau_{1}\left(P S L_{2}(8)\right)=6 \neq 4$, also a contradiction. Thus $G$ is solvable.

Lemma 4.3 Suppose that $G$ is a nonsolvable group with exactly nine classes of nonnormal nontrivial subgroups of the same order, then $G \cong$ $P S L_{2}(7)$ or $S L_{2}(5)$.

Proof. (1) Suppose that $G$ is a nonabelian simple group. By the hypothesis, $G$ has exactly nine classes of nontrivial subgroups of the same order. It 
follows that $G \cong P S L_{2}(7)$ by Theorem 1.6 (4).

(2) Suppose that $G$ is not a simple group. Let $G=G_{0} \triangleright G_{1} \triangleright G_{2} \triangleright$ $\cdots \triangleright G_{i-1} \triangleright G_{i} \triangleright \cdots \triangleright G_{s-1} \triangleright G_{s}=1$ be a composition series from $G$ to $G_{s}$, where $G_{i-1} / G_{i}$ is a simple group for every $1 \leq i \leq s$. Since $G$ is nonsolvable, there exists some $i$ for $1 \leq i \leq s$ such that $G_{i-1} / G_{i}$ is a nonabelian simple group. Let $j$ be the smallest number such that $G_{j-1} / G_{j}$ is a nonabelian simple group. Obviously, $G_{j-1} / G_{j}$ has no normal nontrivial subgroups. By the hypothesis, $G_{j-1} / G_{j}$ has at most nine classes of nontrivial subgroups of the same order. Moreover, by Theorem 1.6, $G_{j-1} / G_{j}$ might have exactly seven or nine classes of nontrivial subgroups of the same order, and then $G_{j-1} / G_{j} \cong A_{5}$ or $P S L_{2}(7)$.

(i) Suppose that $G_{j-1} / G_{j} \cong P S L_{2}(7)$.

(a) Suppose that $G_{j}=1$. It follows that $G_{j-1} \cong P S L_{2}(7)$. Since $G$ is not a simple group, one has $j>1$. By the choice of $j, G_{j-2} / G_{j-1} \cong \mathbb{Z}_{p}$ for some prime $p$. Obviously, $C_{G_{j-2}}\left(G_{j-1}\right) \cap G_{j-1} \unlhd G_{j-1}$. Since $G_{j-1}$ is a nonabelian simple group, one has $C_{G_{j-2}}\left(G_{j-1}\right) \cap G_{j-1}=1$. By N/Ctheorem, $G_{j-2} / C_{G_{j-2}}\left(G_{j-1}\right)=N_{G_{j-2}}\left(G_{j-1}\right) / C_{G_{j-2}}\left(G_{j-1}\right) \lesssim \operatorname{Aut}\left(G_{j-1}\right)=$ $\operatorname{Aut}\left(P S L_{2}(7)\right)=P G L_{2}(7)$. If $C_{G_{j-2}}\left(G_{j-1}\right)=1$. Since $G_{j-2}>G_{j-1}$, one has $G_{j-2} \cong P G L_{2}(7)$. If $C_{G_{j-2}}\left(G_{j-1}\right) \neq 1$, one has $G_{j-2}=$ $G_{j-1} \times C_{G_{j-2}}\left(G_{j-1}\right)$. Then $C_{G_{j-2}}\left(G_{j-1}\right) \cong G_{j-1} C_{G_{j-2}}\left(G_{j-1}\right) / G_{j-1}=$ $G_{j-2} / G_{j-1} \cong \mathbb{Z}_{p}$. It follows that $G_{j-2} \cong P S L_{2}(7) \times \mathbb{Z}_{p}$.

Whenever $G_{j-2} \cong P G L_{2}(7)$ or $P S L_{2}(7) \times \mathbb{Z}_{p}$, one has that $G_{j-2}$ has more than nine classes of nonnormal nontrivial subgroups of the same order, a contradiction.

(b) Suppose that $G_{j} \neq 1$. Assume that $G_{j}$ has non-prime-power order. Then every proper subgroup $H$ of $G_{j-1}$ satisfying $H>G_{j}$ is a nonnormal proper subgroup of $G_{j-1}$ of non-prime-power order. It follows that $G_{j-1}$ has at least nine classes of nonnormal proper subgroups of the same nonprime-power order by Theorem 1.6 (4). Moreover, by Lemma $4.1, G_{j-1}$ has at least four classes of nonnormal nontrivial subgroups of the same primepower order. It follows that $G_{j-1}$ has at least thirteen classes of nonnormal nontrivial subgroups of the same order, a contradiction. Assume that $G_{j}$ has prime-power order. It is easy to see that $G_{j-1}$ has more than five classes of nonnormal proper subgroups of the same non-prime-power order. It also follows that $G_{j-1}$ has more than nine classes of nonnormal nontrivial subgroups of the same order, a contradiction.

(ii) Suppose that $G_{j-1} / G_{j} \cong A_{5}$. 
(c) Suppose that $G_{j}=1$. Then $G_{j-1} \cong A_{5}$. Also, since $G$ is not a simple group, one has $j>1$. By the choice of $j, G_{j-2} / G_{j-1} \cong \mathbb{Z}_{p}$ for some prime $p$. Arguing as in part (i) (a), we can get that $G_{j-2} \cong S_{5}$ or $A_{5} \times \mathbb{Z}_{p}$. However, both $S_{5}$ and $A_{5} \times \mathbb{Z}_{p}$ have more than nine classes of nonnormal nontrivial subgroups of the same order, which implies that $G_{j-2}$ has more than nine classes of nonnormal nontrivial subgroups of the same order, a contradiction.

(d) Suppose that $G_{j} \neq 1$. If $G_{j}$ has non-prime-power order, then every proper subgroup $K$ of $G_{j-1}$ satisfying $K>G_{j}$ is a nonnormal proper subgroup of $G_{j-1}$ of non-prime-power order. It follows that $G_{j-1}$ has at least seven classes of nonnormal proper subgroups of the same non-primepower order by Theorem 1.6. Moreover, by Lemma 4.1, $G_{j-1}$ has at least four classes of nonnormal nontrivial subgroups of the same prime-power order. It follows that $G_{j-1}$ has at least eleven classes of nonnormal nontrivial subgroups of the same order, a contradiction. Next assume that $G_{j}$ has prime-power order. It is easy to see that $G_{j-1}$ has at least six classes of nonnormal proper subgroups of the same non-prime-power order if $\left|G_{j}\right|$ is a $p$-power for $p \geq 3$. Since $G_{j-1}$ must have at least four classes of nonnormal nontrivial subgroups of the same prime-power order, it follows that $G_{j-1}$ has at least ten classes of nonnormal nontrivial subgroups of the same order, a contradiction. Therefore, $\left|G_{j}\right|$ is a 2-power. Assume that $\left|G_{j}\right|=2^{n}$, where $n \geq 1$.

$\left(\mathrm{d}_{1}\right)$ Suppose that $n \geq 2$. If $G_{j}$ has a nontrivial subgroup $G_{t}$ such that $G_{t} \unlhd G_{j-1}$, it is easy to see that $G_{j-1}$ has at least seven classes of nonnormal proper subgroups of the same non-prime-power order, which implies that $G_{j-1}$ has least eleven classes of nonnormal nontrivial subgroups of the same order, a contradiction. If $G_{j}$ has no nontrivial normal subgroups of $G_{j-1}$, it is easy to see that $G_{j-1}$ has at least five classes of nonnormal proper subgroups of the same non-prime-power order. Moreover, arguing as in proof of Lemma 4.1, we can get that $G_{j-1}$ has at least five classes of nonnormal nontrivial subgroups of the same prime-power order. It follows that $G_{j-1}$ has at least ten classes of nonnormal nontrivial subgroups of the same order, also a contradiction.

$\left(\mathrm{d}_{2}\right)$ Suppose that $n=1$. It follows that $G_{j-1}$ might be isomorphic to $S L_{2}(5)$ or $A_{5} \times \mathbb{Z}_{2}$. Obviously, $G_{j-1} ¥ A_{5} \times \mathbb{Z}_{2}$ since $A_{5} \times \mathbb{Z}_{2}$ has more than nine classes of nonnormal nontrivial subgroups of the same order. Therefore, $G_{j-1} \cong S L_{2}(5)$. If $G>G_{j-1}$, then $j>1$. By the choice of $j, G_{j-2} / G_{j-1}$ 
$\cong \mathbb{Z}_{p}$ for some prime $p$. Obviously, $G_{j-2} / G_{j-1} \cong\left(G_{j-2} / Z\left(G_{j-1}\right)\right) /$ $\left(G_{j-1} / Z\left(G_{j-1}\right)\right)$. Since $G_{j-1} / Z\left(G_{j-1}\right) \cong A_{5}$, arguing as in part (i) (a), we can get that $G_{j-2} / Z\left(G_{j-1}\right) \cong S_{5}$ or $A_{5} \times \mathbb{Z}_{p}$, which implies that $G_{j-2}$ has more than nine classes of nonnormal nontrivial subgroups of the same order, a contradiction. Therefore, $G=G_{j-1} \cong S L_{2}(5)$.

Lemmas 4.2 and 4.3 combined together give Theorem 1.7.

Acknowledgements The authors are grateful to the referee who gives valuable comments and suggestions.

\section{References}

[1] Gallian J. A. and Moulton D., The classification of minimal non-primepower order groups. Arch. Math. (Basel), 59 (1992), 521-524.

[2] Shi J. and Zhang C., Finite groups with given quantitative non-nilpotent subgroups. Comm. Algebra, 39 (2011), 3346-3355.

[ 3 ] Shi J. and Zhang C., Finite groups in which the number of classes of nonnilpotent proper subgroups of the same order is given. Chin. Ann. Math. (Ser. A), 32 (2011), 687-692 (in Chinese).

[4] Shi J., Zhang C. and Liang D., Finite groups with some subgroups of given order. Algebra Colloq., 20 (2013), 457-462.

[5] Shi J. and Zhang C., Finite groups with given quantitative non-nilpotent sub-groups II. Comm. Algebra, 42 (2014), 4248-4252. 


\author{
Jiangtao SHI \\ School of Mathematics and Information Science \\ Yantai University \\ Yantai 264005, China \\ E-mail: shijt@math.pku.edu.cn
}

\title{
Cui ZHANG
}

1. School of Mathematics and Information Science Yantai University, Yantai 264005, China

2. University of Primorska FAMNIT, Glagoljaška 8, 6000 Koper, Slovenia E-mail: cuizhang2008@gmail.com

Dengfeng LIANG

Department of Mathematics

Beijing Technology and Business University

Beijing 100048, China

E-mail: dengfengliang@163.com 